\title{
ON INVOLUTIVE CLUSTER AUTOMORPHISMS
}

\author{
NDOUNÉ NDOUNÉ
}

\begin{abstract}
We construct a special embedding of the translation quiver $\mathbb{Z} Q$ in the three-dimensional affine space $\mathbb{R}^{3}$ where $Q$ is a finite connected acyclic quiver and $\mathbb{Z} Q$ contains a local slice whose quiver is isomorphic to the opposite quiver $Q^{o p}$ of $Q$. Via this embedding, we show that there exists an involutive anti-automorphism of the translation quiver $\mathbb{Z} Q$. As an immediate consequence, we characterize explicitly the group of cluster automorphisms of the cluster algebras of seed $(X, Q)$, where $Q$ and $Q^{o p}$ are mutation equivalent.
\end{abstract}

\section{INTRODUCTION}

Cluster algebras were invented by Fomin and Zelevinsky [11 in order to understand the canonical bases of quantized enveloping algebras associated to semi-simple Lie algebras [18. A cluster algebra is a commutative ring with a distinguished set of generators, called cluster variables. The set of all cluster variables is constructed recursively from an initial set using a procedure called mutation.

Today the theory of cluster algebras relates with numerous fields of mathematics, for example, representation theory of algebras, Lie theory, Poisson geometry, Teichmüller theory and integrable systems. Following 3 2, we are interested in studying the involutive cluster automorphisms. The objective of this paper is to solve an open problem posed in [3]; it was conjectured that if $Q$ is a finite connected acyclic quiver which is mutation equivalent to $Q^{o p}$, then the group of cluster automorphisms of the cluster algebra of a seed $(X, Q)$ is a semi-direct product of the subgroup of the direct cluster automorphisms with $\mathbb{Z}_{2}$. A partial answer was given in [3], in the case when the underlying graph of $Q$ is a tree. We prove it here in general.

In this paper, we construct an embedding of a repetitive translation quiver $\mathbb{Z} Q$ in 3 -space $\mathbb{R}^{3}$, when $Q$ is a finite connected acyclic quiver and the translation quiver contains a local slice whose quiver is isomorphic to $Q^{o p}$. In particular, we construct an embedding of the transjective component of the Auslander Reiten quiver of the cluster category $\mathcal{C}_{Q}$. We then use this construction to prove the existence of an involutive inverse cluster automorphism of a cluster algebra with a seed $(X, Q)$, where $Q$ is a finite connected acyclic quiver which is mutation equivalent to $Q^{o p}$. 
Our paper is organized as follows. After an introductory section 2 , we construct in section 3 , the required embedding $\eta$ of $\mathbb{Z} Q$ in $\mathbb{R}^{3}$, such that if $\Sigma$ and $\widetilde{\Sigma}$ are two local slices whose quivers are respectively isomorphic to $Q$ and $Q^{o p}$, then $\eta(\Sigma)$ and $\eta(\widetilde{\Sigma})$ are symmetric with respect to a particular plane.

In section 4 , we prove that, if $Q$ is a finite acyclic quiver which is mutation equivalent to $Q^{o p}$, then the group of cluster automorphisms of a cluster algebra of a seed $(X, Q)$ is a semi-direct product of the subgroup of the direct cluster automorphisms with $\mathbb{Z}_{2}$.

\section{ACKNOWLEDGMENTS}

This article is part of my $\mathrm{PhD}$ thesis, under the supervision of Ibrahim Assem and Vasilisa Shramchenko. I would like to thank them deeply for their patience and availability.

\section{Cluster Automorphisms}

The notion of cluster automorphisms was introduced in [3] and it is connected with quiver automorphisms. We recall that a quiver is a quadruple $Q=\left(Q_{0}, Q_{1}, s, t\right)$ consisting of two sets, $Q_{0}$ (whose elements are called vertices) and $Q_{1}$ (whose elements are called arrows), and of two maps $s, t: Q_{1} \longrightarrow Q_{0}$ associating to each arrow $\alpha \in Q_{1}$ its source $s(\alpha)$ and its target $t(\alpha)$. If $i=s(\alpha)$ and $j=t(\alpha)$, we denote this situation by $i \stackrel{\alpha}{\longrightarrow} j$. Given a vertex $i$, we set $i^{+}=\left\{\alpha \in Q_{1} \mid s(\alpha)=i\right\}$ and $i^{-}=\left\{\alpha \in Q_{1} \mid t(\alpha)=i\right\}$.

Let $Q$ be a finite connected quiver without oriented cycles of length one or two. Let $n=\left|Q_{0}\right|$ denote the number of vertices in $Q, X=$ $\left\{x_{1}, x_{2}, \ldots, x_{n}\right\}$ be a set of $n$ variables and denote the vertices by $Q_{0}=$ $\{1,2, \ldots, n\}$, where we agree that the point $i$ corresponds to the variable $x_{i}$. We consider the field $\mathcal{F}=\mathbb{Q}\left(x_{1}, x_{2}, \ldots, x_{n}\right)$ of rational functions in $x_{1}, x_{2}, \ldots, x_{n}$ (with rational coefficients). The cluster algebra $\mathcal{A}=\mathcal{A}(X, Q)$ is the $\mathbb{Z}$-subalgebra of $\mathcal{F}$ defined by a set of generators obtained recursively from $X$ in the following manner. Let $k$ be such that $1 \leq k \leq n$. We define the mutation $\mu_{x_{k}, X}$ (or $\mu_{x_{k}}$ or $\mu_{k}$ for brevity if there is no ambiguity) of the pair $(X, Q)$ to be a new pair $\left(X^{\prime}, Q^{\prime}\right)$, that is $\mu_{k}(X, Q)=\left(X^{\prime}, Q^{\prime}\right)$, where $Q^{\prime}$ is the quiver obtained from $Q$ by performing the following operations:

(a) for any path $i \longrightarrow k \longrightarrow j$ of length two having $k$ as mid-vertex, we insert a new arrow $i \longrightarrow j$.

(b) all arrows incident to the vertex $k$ are reversed,

(c) all cycles of length two are deleted.

On the other hand, $X^{\prime}$ is a set of variables defined as follows. $X^{\prime}=\left(X \backslash\left\{x_{k}\right\}\right) \cup\left\{x_{k}^{\prime}\right\}$ where $x_{k}^{\prime} \in \mathcal{F}$ is obtained from $X$ by the 
so-called exchange relation:

$$
x_{k} x_{k}^{\prime}=\prod_{\alpha \in i^{+}} x_{t(\alpha)}+\prod_{\alpha \in i^{-}} x_{s(\alpha)} .
$$

Let $\mathcal{X}$ be the union of all possible sets of variables obtained from $X$ by successive mutations. Then the cluster algebra $\mathcal{A}=\mathcal{A}(X, Q)$ is the $\mathbb{Z}$-subalgebra of $\mathcal{F}$ generated by $\mathcal{X}$.

Each pair $(\tilde{X}, \tilde{Q})$ obtained from $(X, Q)$ by successive mutations is called a seed, and the set $\tilde{X}$ is called a cluster. The elements $\tilde{x}_{1}, \tilde{x}_{2}, \ldots, \tilde{x}_{n}$ of a cluster $\tilde{X}$ are called cluster variables. Each cluster is a transcendence basis for the field $\mathcal{F}$. The pair $(X, Q)$ is called the initial seed, and $X$ is called the initial cluster.

Gekhtman, Shapiro and Vainshtein showed in 13 that for every seed $(\tilde{X}, \tilde{Q})$ the quiver $\tilde{Q}$ is uniquely defined by the cluster $\tilde{X}$, and we use the notation $Q(\tilde{X})$ for the quiver of a given cluster $\tilde{X}$. We write $p_{x}$ for the point of $Q(\tilde{X})$ corresponding to the cluster variable $x \in \tilde{X}$. Following [11] we recall that the Laurent phenomenon asserts that each cluster variable in $\mathcal{A}$ can be expressed as a Laurent polynomial in $x_{i}$, with $1 \leq i \leq n$, that is, every cluster variable is of the form

$$
\frac{p\left(x_{1}, x_{2}, \ldots, x_{n}\right)}{\prod_{l=1}^{n} x_{l}^{d_{l}}},
$$

where $p \in \mathbb{Z}\left[x_{1}, x_{2}, \ldots, x_{n}\right]$, and $d_{l}$ is a positive integer(for $1 \leq l \leq n$ ).

As a consequence, $\mathcal{A} \subseteq \mathbb{Z}\left[x_{1}^{ \pm 1}, \ldots, x_{n}^{ \pm 1}\right]$, see [11] for more details. The following definition is due to 3 .

Definition 2.1. Let $\mathcal{A}$ be a cluster algebra, and let $f: \mathcal{A} \longrightarrow \mathcal{A}$ be an automorphism of $\mathbb{Z}$-algebras. Then $f$ is called cluster automorphism if there exists a seed $(X, Q)$ of $\mathcal{A}$ such that the following conditions are satisfied:

(CA1) $f(X)$ is a cluster;

(CA2) $f(X)$ is compatible with mutations, that is, for every $x \in X$, we have $f\left(\mu_{x, X}(x)\right)=\mu_{f(x), f(X)}(f(x))$.

Now we state an equivalent characterization of cluster automorphisms see [3,Lemma 2.3].

Lemma 2.1. Let $f$ be a $\mathbb{Z}$-algebra automorphism of $\mathcal{A}$. Then $f$ is a cluster automorphism if and only if there exists a seed $(X, Q)$ such that $f(X)$ is a cluster and one of the following two conditions is satisfied: (a) there exists an isomorphism of quivers $\varphi: Q \longrightarrow Q(f(X))$ such that $\varphi\left(p_{x}\right)=p_{f(x)}$ for all $p_{x} \in Q_{0}$, or

(b) there exists an isomorphism of quivers $\varphi: Q^{\text {op }} \longrightarrow Q(f(X))$ such that $\varphi\left(p_{x}\right)=p_{f(x)}$ for all $p_{x} \in Q_{0}$. 
A cluster automorphism $f$ is called direct if it satisfies condition $(a)$ of the above lemma, and $f$ is called inverse if it satisfies condition $(b)$.

The set of all cluster automorphisms forms a group under composition denoted by $\operatorname{Aut}(\mathcal{A})$ and called the group of cluster automorphisms. The set of all direct automorphisms is denoted by $\operatorname{Aut}^{+}(\mathcal{A})$; it is a normal subgroup of $\operatorname{Aut}(\mathcal{A})$ of index at most two. For more results and properties of cluster automorphisms, we refer the reader to 3 .

\section{INVOLUTIVE AUTOMORPHISMS OF QUIVERS}

In this section, we assume that $Q$ is a finite connected acyclic quiver. Let $K$ be an algebraically closed field, $K Q$ the path algebra of $Q$ and $\bmod K Q$ the category of finitely generated right $K Q$-modules. For the properties of $\bmod K Q$ and its Auslander-Reiten quiver $\Gamma(\bmod K Q)$ we refer the reader to [5, 4]. We denote by $\mathcal{D}^{d}(\bmod K Q)$ the bounded derived category over $\bmod K Q$. This is a triangulated Krull-Schmidt category having a Serre duality and hence almost split triangles. Since $K Q$ is hereditary, the Auslander Reiten quiver $\Gamma\left(\mathcal{D}^{d}(\bmod K Q)\right)$ of $\mathcal{D}^{d}(\bmod K Q)$ is well-understood, see [14].

Following [4] we give the definitions and some basic properties of a well-known translation quiver. If $Q$ is a connected acyclic quiver, the repetitive quiver $\mathbb{Z} Q$ is the quiver whose set of vertices is $(\mathbb{Z} Q)_{0}=$ $\left\{(m, \nu) \mid m \in \mathbb{Z}, \nu \in Q_{0}\right\}$ and the set of arrows is $(\mathbb{Z} Q)_{1}=\{(m, \alpha)$ : $\left.(m, \nu) \rightarrow\left(m, \nu^{\prime}\right) \mid m \in \mathbb{Z}, \alpha: \nu \rightarrow \nu^{\prime}\right\} \cup\left\{\left(m, \alpha^{\prime}\right):\left(m, \nu^{\prime}\right) \rightarrow(m-\right.$ $\left.1, \nu) \mid m \in \mathbb{Z}, \alpha: \nu \rightarrow \nu^{\prime}\right\}$. The map $\tau: \mathbb{Z} Q \longrightarrow \mathbb{Z} Q$ defined by $\tau(m, \nu)=(m-1, \nu)$ is called the translation of the quiver $\mathbb{Z} Q$. The pair $(\mathbb{Z} Q, \tau)$ is a translation quiver, for more details we refer to [4]. The translation quiver $\mathbb{Z} Q$ is stable because its translation is defined everywhere. We recall that an automorphism of a translation quiver is a quiver automorphism commuting with the translation $\tau$.

The quiver $\Gamma\left(\mathcal{D}^{d}(\bmod K Q)\right)$ consists of two types of components: the regular components and the transjective components, the latter are of the form $\mathbb{Z} Q$, see 14 . The cluster category $\mathcal{C}_{Q}$ of Buan-Marsh-ReinekeReiten-Todorov is defined to be the orbit category of $\mathcal{D}^{d}(\bmod K Q)$ under the action of the automorphism $\tau^{-1}[1]$, where $\tau$ is the AuslanderReiten translation and [1] is the shift of $\mathcal{D}^{d}(\bmod K Q)$, see [7]. Then $\mathcal{C}_{Q}$ is also a triangulated Krull-Schmidt category having almost split triangles. Moreover, $\mathcal{C}_{Q}$ is a 2-Calabi-Yau category [7]. The AuslanderReiten quiver $\Gamma\left(\mathcal{C}_{Q}\right)$ of $\mathcal{C}_{Q}$ is the quotient of $\Gamma\left(\mathcal{D}^{d}(\bmod K Q)\right)$ under the action of the quiver automorphism induced by $\tau^{-1}[1]$. Then, the combinatorics of cluster variables is encoded in the cluster category. That is, if $\mathcal{A}=\mathcal{A}(X, Q)$ is a cluster algebra, with $Q$ an acyclic quiver having $n=\left|Q_{0}\right|$ points, there exists a map $X_{?}: \mathcal{C}_{Q} \longrightarrow \mathbb{Z}\left[x_{1}^{ \pm 1}, \ldots, x_{n}^{ \pm 1}\right]$ called the canonical cluster character, or the Caldero-Chapoton map. The map $X_{\text {? }}$ induces a bijection between the indecomposables $M$ (up to isomorphism) in $\mathcal{C}_{Q}$ which have no self-extensions and the cluster 
variables $X_{M}$, see [8]. Under this bijection, clusters correspond to cluster-tilting objects in $\mathcal{C}_{Q}$.

It has been shown in [3] that, if $\mathcal{A}$ is a cluster algebra with a seed $(X, Q)$ such that the underlying graph of $Q$ is a tree, then there exists an inverse cluster automorphism $\sigma \in \operatorname{Aut}(\mathcal{A})$ of order two and therefore $\operatorname{Aut}(\mathcal{A})=\operatorname{Aut}^{+}(\mathcal{A}) \rtimes \mathbb{Z}_{2}$. In this section we show that this statement is valid for any acyclic quiver which is mutation equivalent to its opposite quiver.

Let $j$ be an integer, a subquiver of $\mathbb{Z} Q$ of the form $(j, Q)$ is a full subquiver of $\mathbb{Z} Q$ whose set of vertices is $(j, Q)_{0}=\left\{(j, \nu) \mid \nu \in Q_{0}\right\}$.

We denote by $C^{j}$ the subquiver of the quiver $\mathbb{Z} Q$ of the form $(j, Q)$.

The notion of local slice was introduced in 1 for the connected components of Auslander-Reiten quivers of the categories of modules. For the repetitive quiver $\mathbb{Z} Q$, the notion of local slice coincides with the notion of section as defined in [4, p.302].

We consider the 3 -dimensional Euclidean space $\mathbb{R}^{3}$ with coordinates $(x, y, z)$, where the frame of reference has a negative orientation as shown in Fig 1.

Our main result will be obtained as a corollary of the following theorem.

Theorem 3.1. Let $Q$ be a finite connected acyclic quiver such that the translation quiver $\mathbb{Z} Q$ contains two local slices $\Sigma$ and $\widetilde{\Sigma}$ whose quivers are respectively isomorphic to $Q$ and $Q^{o p}$. Then there is an embedding $\eta: \mathbb{Z} Q \longrightarrow \mathbb{R}^{3}$ and an oblique reflection with respect to the plane $(x O z)$ which maps $\eta(\Sigma)$ and $\eta(\widetilde{\Sigma})$ to each other.

Proof. The translation quiver $\mathbb{Z} Q$ contains two local slices $\Sigma$ and $\widetilde{\Sigma}$ whose quivers are isomorphic respectively to $Q$ and $Q^{o p}$. Since the translation quiver $\mathbb{Z} Q$ is stable, we can assume without loss of generality that $\Sigma$ and $\widetilde{\Sigma}$ are such that $\Sigma \cap \widetilde{\Sigma} \neq \emptyset$ and $\Sigma$ is chosen to be the copy of $Q$ at zero, that is $\Sigma=(0, Q)=C^{0}$.

Because $\widetilde{\Sigma}$ is a subquiver of $\mathbb{Z} Q$ and $\Sigma_{0} \cap \widetilde{\Sigma}_{0} \subset C^{0}$, there exists a maximal nonnegative integer $k$, such that $C^{-j} \cap \widetilde{\Sigma} \neq \emptyset$ for all $j$ with $0 \leq j \leq k$. We use the following notation for the vertices of $\Sigma$ : the vertex $\left(0, \nu_{i}\right)$ is denoted by $\left(0, \nu_{j i}\right)$, where $j$ is such that the vertex $\left(-j, \nu_{i}\right)$ belongs to $\widetilde{\Sigma}$, for $0 \leq j \leq k$. We extend this notation to the translation quiver $\mathbb{Z} Q$ that is $\left(-m, \nu_{j i}\right)=\tau^{m}\left(0, \nu_{j i}\right)$. We denote by $s_{j}$, where $0 \leq j \leq k$, the number of vertices $\left(0, \nu_{j i}\right)$. Thus we have $\sum_{j=0}^{k} s_{j}=\left|Q_{0}\right|$.

The proof is completed in the following three steps $(a)(b)(c)$.

(a) If $A_{1}, A_{2}, \ldots, A_{h}$ are points of $\mathbb{R}^{3}$, we denote by $\left[A_{1} A_{2} \ldots A_{h}\right]$ the convex hull of the points $A_{1}, A_{2}, \ldots, A_{h}$. 


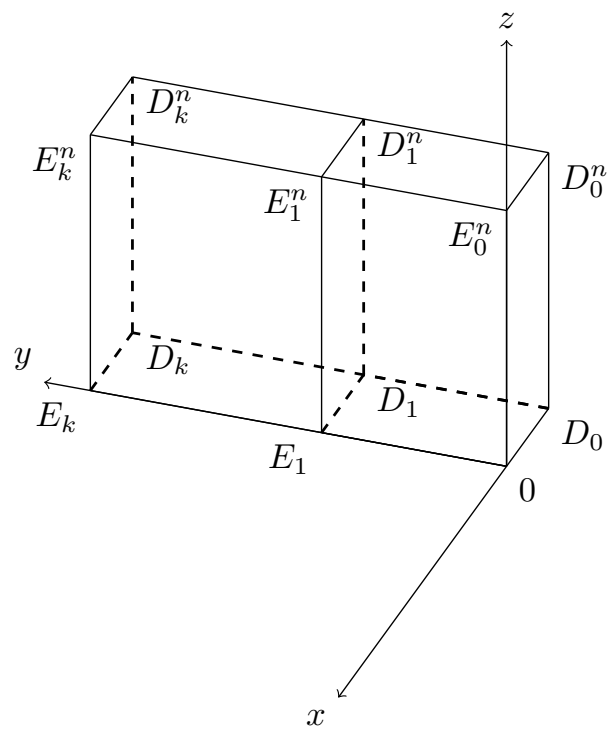

Fig.1

Let $n=\left|Q_{0}\right|$ and define $\mathbb{P}_{n k}$ to be the convex hull of the points $O, D_{0}, D_{k}, E_{k}, E_{0}^{n}, E_{k}^{n}, D_{0}^{n}, D_{k}^{n}$ whose coordinates are given by:

$O=(0,0,0), D_{0}=(-1,0,0), D_{k}=(-1, k, 0), E_{k}=(0, k, 0), E_{0}^{n}=$ $(0,0, n), E_{k}^{n}=(0, k, n), D_{0}^{n}=(-1,0, n), D_{k}^{n}=(-1, k, n)$; then $\mathbb{P}_{n k}$ is a rectangular parallelepiped (see Fig.1).

We want to embed $\Sigma$ in $\mathbb{R}^{3}$ in such a way that the vertices of the embedded quiver $\eta(\Sigma)$ belong to $\mathbb{P}_{n k} \backslash\left[D_{0} D_{k} D_{k}^{n} D_{0}^{n}\right]$ that is, to the parallelepiped $\mathbb{P}_{n k}$ from which we remove the face $D_{0} D_{k} D_{k}^{n} D_{0}^{n}$. We set $H=(0,0, n-1)$ and $T=(-1,0, n-1)$. Let $\left(\mathcal{P}_{m}\right)_{m \in \mathbb{Z}}$ be the family of parallel planes in $\mathbb{R}^{3}$ defined by the equations $\mathcal{P}_{m}: y=m$. We decompose $\Sigma$ into $k+1$ non-intersecting subquivers $\Sigma^{j}$ and construct the embedding of each subquiver separately.

The construction of $\eta(\Sigma)$ is thus done in three steps $\left(a_{i}\right),\left(a_{i i}\right)$ and $\left(a_{i i i}\right)$ as follows.

$\left(a_{i}\right)$ Construction of $\eta\left(\Sigma^{0}\right)$, where $\Sigma^{0}=\Sigma \cap \widetilde{\Sigma}$. Then $\Sigma^{0}$ is the full subquiver of $\Sigma$ whose set of vertices is $\Sigma_{0}^{0}=\left\{\left(0, \nu_{0 l}\right) \mid 1 \leq l \leq s_{0}\right\}$. We map the vertices $\left(0, \nu_{01}\right),\left(0, \nu_{02}\right), \ldots,\left(0, \nu_{0 s_{0}}\right)$ of $\Sigma$, to distinct points $A_{01}$, $A_{02}, \ldots, A_{0 s_{0}}$ in $\left[E_{0}^{n} D_{0}^{n} T H\right] \backslash\left[T D_{0}^{n}\right]$ such that for all $i, j \in\left\{1,2, \ldots, s_{0}\right\}$ the line $\left(A_{0 i} A_{0 j}\right)$ is not parallel to a line $\left(O D_{0}\right)$. Each arrow $\alpha$ of $\Sigma$ from the vertex $\left(0, \nu_{0 i}\right)$ to the vertex $\left(0, \nu_{0 j}\right)$ is mapped to an arrow $\bar{\alpha}$ from $A_{0 i}$ to $A_{0 j}$. In other words, we set $\eta\left(0, \nu_{0 i}\right)=A_{0 i}$ with $1 \leq i \leq s_{0}$ and $\eta(\alpha)=\bar{\alpha}$. The quiver $\eta\left(\Sigma^{0}\right)$ obtained in the plane $\mathcal{P}_{0}$ is isomorphic to $\Sigma^{0}$, we call it an embedding of $\Sigma^{0}$.

$\left(a_{i i}\right)$ Now we construct $\eta\left(\Sigma^{1}\right)$, where $\Sigma^{1}$ is the full subquiver of $\Sigma$ whose set of vertices is $\Sigma_{0}^{1}=\left\{\left(0, \nu_{1 l}\right) \mid 1 \leq l \leq s_{1}\right\}$.

We denote by $t_{\vec{v}}$ the translation in $\mathbb{R}^{3}$ by the vector $\vec{v}=(0,1,-1)$. 
Let $\Sigma_{0}^{11}$ be the set of all vertices $\left(0, \nu_{1 l}\right)$ of $\Sigma_{0}^{1}$ such that there exists at least one arrow from a vertex $\left(0, \nu_{0 i}\right)$ in $\Sigma^{0}$ to the vertex $\left(-1, \nu_{1 l}\right)$. And denote by $\Sigma_{0}^{12}$ the complement of $\Sigma_{0}^{11}$ in $\Sigma_{0}^{1}$; then $\Sigma_{0}^{12}$ is the set of the elements $\left(0, \nu_{1 l}\right)$ in $\Sigma_{0}^{1}$ such that there is no arrow from $\Sigma^{0}$ to $\left(-1, \nu_{1 l}\right)$. Since the quiver $\Sigma$ is connected, we have $\Sigma_{0}^{11} \neq \emptyset$.

For the construction of the embedding of the set $\Sigma_{0}^{11}$, we divide $\Sigma_{0}^{11}$ into two families of subsets as follows.

The first family consists of the non-intersecting subsets $L_{1}, L_{2}, \ldots, L_{h}$ of $\Sigma_{0}^{11}$ such that $\left|L_{j}\right| \geq 2$ and for some $\left(0, \nu_{0 i_{j}}\right) \in \Sigma_{0}^{0}$ there exists an arrow to $\left(0, \nu_{0 i_{j}}\right)$ from each element $L_{j}$. The second family is given by the remaining elements of $\Sigma_{0}^{11}$, the elements which do not belong to any set $L_{j}$ with $1 \leq j \leq h$.

Now we construct first the embedding of the elements of the set $\Sigma_{0}^{11} \backslash\left(L_{1} \cup L_{2} \ldots \cup L_{h}\right)$. For an element $\left(0, \nu_{1 r}\right)$ of $\Sigma_{0}^{11} \backslash\left(L_{1} \cup L_{2} \ldots \cup L_{h}\right)$, we take any arrow originating in $\left(0, \nu_{1 r}\right)$ whose target is $\left(0, \nu_{0 i_{r}}\right) \in \Sigma_{0}^{0}$. Then the vertex $\left(0, \nu_{1 r}\right)$ is mapped to the point $A_{1 r}:=t_{\vec{v}}\left(A_{0 i_{r}}\right)$.

For the construction of the embedding of the elements of the set $L_{j}$ with $1 \leq j \leq h$, we choose an element $\left(0, \nu_{1 l_{j}}\right)$ in $L_{j}$ and we map it to a point $A_{1 l_{j}}:=t_{\vec{v}}\left(A_{0 i_{j}}\right)$ with $A_{0 i_{j}}=\eta\left(0, \nu_{0 i_{j}}\right)$. In order to define the embedding for the remaining elements of $L_{j}$, we proceed as follows. Let $\Delta_{j}$ be the line parallel to $(O x)$ passing through $A_{1 l_{j}}$ and let $[F G]$ be the intersection of $\Delta_{j}$ and the rectangle $\left[E_{1} D_{1} D_{1}^{n} E_{1}^{n}\right]$ (we recall that $\left.A_{1 l_{j}} \in\left[E_{1} D_{1} D_{1}^{n} E_{1}^{n}\right]\right)$. The elements of $L_{j} \backslash\left\{\left(0, \nu_{1 l_{j}}\right)\right\}$ are mapped to arbitrary distinct points in $[G F] \backslash\left\{G, F, A_{1 l_{j}}\right\}$. The elements of $L_{i}$, $i \neq j, 1 \leq i \leq h$ are mapped to the distinct point of the line $\Delta_{i}$ constructed as above. We have $\Delta_{i} \cap \Delta_{j}=\emptyset$, for $i \neq j$.

It remains to construct the elements of the set $\Sigma_{0}^{12}$. We map the elements of $\Sigma_{0}^{12}$ to distinct points (distinct from the points associated with the elements of $\left.\Sigma_{0}^{11}\right)$ in $t_{\vec{v}}\left(\left[E_{0}^{n} D_{0}^{n} T H\right] \backslash\left[T D_{0}^{n}\right]\right)$ such that any line containing at least two of them is not parallel to $\left(O D_{0}\right)$.

Each arrow $\alpha$ of $\Sigma$ from the vertex $\left(0, \nu_{1 r}\right) \in \Sigma_{0}^{12}$ to the vertex $\left(0, \nu_{1 l}\right) \in \Sigma_{0}^{1}$ is mapped to an arrow $\bar{\alpha}$ from $A_{1 r}=\eta\left(0, \nu_{1 r}\right)$ to $A_{1 l}=$ $\eta\left(0, \nu_{1 l}\right)$ and each arrow $\beta$ of $\Sigma$ from the vertex $\left(0, \nu_{1 j}\right) \in \Sigma_{0}^{11}$ to the vertex $\left(0, \nu_{0 i}\right) \in \Sigma_{0}^{0}$ is mapped to an arrow $\bar{\beta}$ from $A_{1 j}=\eta\left(0, \nu_{1 j}\right)$ to $A_{0 i}=\eta\left(0, \nu_{1 i}\right)$.

The obtained quiver $\eta\left(\Sigma^{1}\right)$ is an embedding of the subquiver $\Sigma^{1}$ into the plane $\mathcal{P}_{1}: y=1$. More precisely, $\eta\left(\Sigma^{1}\right)$ is constructed in the rectangle $\mathcal{P}_{1} \cap \mathbb{P}_{n k}$.

Thus, we have constructed the quiver $\eta\left(\overline{\Sigma^{0} \cup \Sigma^{1}}\right)$, where $\overline{\Sigma^{0} \cup \Sigma^{1}}$ is the full subquiver of $\Sigma$ whose set of vertices is $\Sigma_{0}^{0} \cup \Sigma_{0}^{1}$.

$\left(a_{i i i}\right)$ Construction of $\eta\left(\Sigma^{j}\right)$, where $\Sigma^{j}, 2 \leq j \leq k$ is the full subquiver of $\Sigma$ whose set of vertices is $\Sigma_{0}^{j}=\left\{\left(0, \nu_{j l}\right) \mid 1 \leq l \leq s_{j}\right\}$.

We construct $\eta\left(\Sigma^{j}\right)$ assuming that $\eta\left(\Sigma^{j-1}\right)$ is constructed.

We reproduce the construction of step $a_{(i i)}$ with $\Sigma^{j}$ playing the role of $\Sigma^{1}$ and $\Sigma^{j-1}$ playing the role of $\Sigma^{0}$. The vertices of $\Sigma^{j}$ are mapped by 
$\eta$ to the rectangle $\left[E_{j} D_{j} D_{j}^{n} E_{j}^{n}\right] \backslash\left[D_{j} D_{j}^{n}\right]$ and the arrows between two vertices $\nu$ and $\zeta$ of $\Sigma_{0}$ are mapped to arrows between the corresponding images $\eta(\nu)$ and $\eta(\zeta)$. Therefore, we construct the embedding $\eta\left(\Sigma^{j}\right)$ of $\Sigma^{j}$ into the plane $\mathcal{P}_{j}: y=j$ for $0 \leq j \leq k$, more precisely $\eta\left(\Sigma^{j}\right) \in$ $\mathcal{P}_{j} \cap \mathbb{P}_{n k}$. Thus, we have constructed the quiver $\eta\left(\overline{\Sigma^{0} \cup \Sigma^{1} \ldots \cup \Sigma^{j}}\right)$, where $\overline{\Sigma^{0} \cup \Sigma^{1} \ldots \cup \Sigma^{j}}$ is the full subquiver of $\Sigma$ whose set of vertices is $\Sigma_{0}^{0} \cup \Sigma_{0}^{1} \ldots \cup \Sigma_{0}^{j}$. In other words, we have constructed the embedding of $\overline{\Sigma^{0} \cup \Sigma^{1} \ldots \cup \Sigma^{j}}$ in $\left[O D_{0} D_{0}^{n} E_{0}^{n} E_{j}^{n} E_{j} D_{j} D_{j}^{n}\right] \backslash\left[D_{0} D_{0}^{n} D_{j}^{n} D_{j}\right]$.

Since $\Sigma$ is finite, the process ends after $k$ iterations and we find the required embedded quiver $\eta(\Sigma)$. By construction we have $\eta(\Sigma)=$ $\eta\left(\overline{\Sigma^{0} \cup \Sigma^{1} \ldots \cup \Sigma^{k}}\right)$ and $\left|\eta(\Sigma)_{0}\right|=\left|\Sigma_{0}\right|$. Also, we have an isomorphism of quivers $\eta(\Sigma) \cong \Sigma$ and $\eta(\Sigma)$ is an embedding of $\Sigma$ in $\left[O D_{0} D_{0}^{n} E_{0}^{n} E_{1}^{n} E_{1} D_{1} D_{1}^{n}\right] \backslash$ $\left[D_{0} D_{0}^{n} D_{1}^{n} D_{1}\right]$.

(b) Construction of the quiver $\eta(\mathbb{Z} Q)$ in $\mathbb{R}^{3}$. To define $\eta(\mathbb{Z} Q)$ in $\mathbb{R}^{3}$, we need first to construct each $\eta\left(C^{m}\right)$, with $m \in \mathbb{Z}$, where $C^{m}$ is a copy of the quiver $Q$.

Let $\vec{u}=(-1,-2,0)$ and $t_{\vec{u}}$ be the translation in $\mathbb{R}^{3}$ by vector $\vec{u}$.

We extend $\eta$ to each copy $C^{m}$, with $m \in \mathbb{Z}$, by $\eta\left(C^{m}\right)=t_{m \vec{u}}(\eta(\Sigma))$. We complete the construction of $\eta(\mathbb{Z} Q)$ as one would do for the repetitive quiver $\mathbb{Z} Q$ using that $\eta(\tau)=t_{\vec{u}}$. By the construction, there are no arrows which cross each other when considered as segments in $\mathbb{R}^{3}$. Thus we have extended the embedding $\eta$ to $\mathbb{Z} Q$.

(c) Finally we show that $\eta(\widetilde{\Sigma})$ is the image of $\eta(\Sigma)$ under the oblique reflection $S$ with respect to the plane $\mathcal{P}_{0}$, and the direction given by $\vec{u}$.

If $A=(a, b, c)$ is a point of $\mathbb{R}^{3}$, then the image of $A$ under the oblique reflection $S$ is the point $S(A)=(a-b,-b, c)$.

Let $A_{j r}=\left(a_{j r}, b_{j r}, c_{j r}\right)$ be a point of $\eta(\Sigma)$ such that $\eta\left(0, \nu_{j r}\right)=A_{j r}$, with $\left(0, \nu_{j r}\right) \in \Sigma_{0}$. Since $A_{j r} \in \mathcal{P}_{j}$, then the second coordinate of $A_{j r}$ is equal to $j$, and $S\left(A_{j r}\right)=\left(a_{j r}-j,-j, c_{j r}\right)$.

By the definition of $\eta$, we have $\eta(\tau)=t_{\vec{u}}$ and $\eta\left(\tau^{-1}\right)=t_{-\vec{u}}$.

Now we want to show that $\eta(\widetilde{\Sigma})=S(\eta(\Sigma))$.

Let $N_{r} \in \widetilde{\Sigma}_{0}$, then $N_{r}=\left(-j, \nu_{j r}\right)=\tau^{j}\left(0, \nu_{j r}\right)$, with $1 \leq r \leq s_{j}$, where $\sum_{j=0}^{k} s_{j}=n$. We have

$$
\begin{aligned}
\eta\left(N_{r}\right) & =\eta\left(\tau^{j}\left(0, \nu_{j r}\right)\right) \\
& =t_{j \vec{u}}\left(A_{j r}\right) \\
& =\left(a_{j r}-j,-j, c_{j r}\right) \\
& =S\left(A_{j r}\right) .
\end{aligned}
$$

Therefore $\eta\left(\widetilde{\Sigma}_{0}\right) \subseteq S\left(\eta\left(\Sigma_{0}\right)\right)$. Since $\left|\eta\left(\widetilde{\Sigma}_{0}\right)\right|=\left|S\left(\eta\left(\Sigma_{0}\right)\right)\right|$, then $\eta\left(\widetilde{\Sigma}_{0}\right)=$ $S\left(\eta\left(\Sigma_{0}\right)\right)$. Because the quivers $\Sigma$ and $\widetilde{\Sigma}$ are full subquivers of $\mathbb{Z} Q$, it follows that $S(\eta(\Sigma))$ and $\eta(\widetilde{\Sigma})$ are also full subquivers of $\eta(\mathbb{Z} Q)$. Thus $S(\eta(\Sigma))=\eta(\widetilde{\Sigma})$. This completes the proof. 
Definition 3.1. The quiver $\eta(\mathbb{Z} Q)$ is called a spatial representation of $\mathbb{Z} Q$ in $\mathbb{R}^{3}$.

Remark 3.1. The reflection $S$ defined in $\mathbb{R}^{3}$ in step (c) of the proof transforming $\eta(\Sigma)$ to $\eta\left(\Sigma^{o p}\right)$ does not induce necessarily a quiver morphism, as shown in the following example.

Example 3.1. Let $Q$ be a quiver defined by:

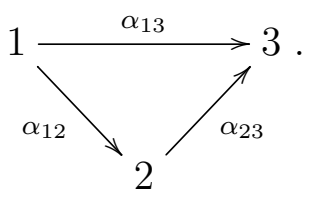

The quiver $\mathbb{Z} Q$ contains a local slice $\widetilde{\Sigma}$ isomorphic to $Q^{\text {op }}$ and that intersects the local slice $\Sigma=(0, Q)$. The local slice $\Sigma$ is given by

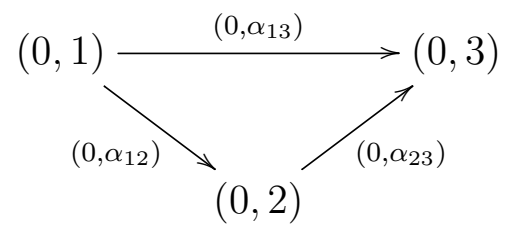

and the local slice $\widetilde{\Sigma}$ is given by

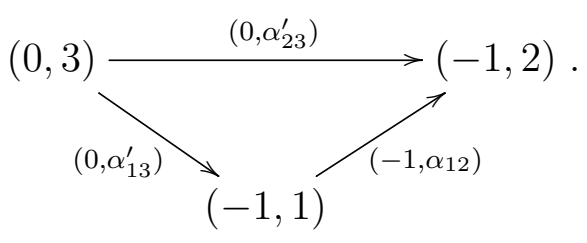

We set $\eta(0,3)=(0,0,2), \eta(0,2)=(0,1,1)$ and $\eta(0,1)=(0,1,0)$. We have $S(\eta(0,3))=(0,0,2), S(\eta(0,2))=\eta(-1,2)$ and $S(\eta(0,1))=$ $\eta(-1,1)$. The reflection $S$ maps the arrow $\eta\left(0, \alpha_{12}\right): \eta(0,1) \rightarrow \eta(0,2)$ to the arrow $\eta\left(-1, \alpha_{12}\right): \eta(-1,1) \rightarrow \eta(-1,2)$, with $S(\eta(0,1))=\eta(-1,1)$ and $S(\eta(0,2))=\eta(-1,2)$. Thus $S$ does not induce an anti-morphism. The reflection $S$ does not induce a quiver morphism, because for the arrow $\eta\left(0, \alpha_{23}\right): \eta(0,2) \rightarrow \eta(0,3)$ of $\eta(\Sigma)$, we have $S\left(\eta\left(0, \alpha_{23}\right)\right)=$ $\eta\left(0, \alpha_{23}^{\prime}\right): \eta(0,3) \rightarrow \eta(-1,2)$.

\section{INVOLUTIVE CLUSTER AUTOMORPHISMS}

Before giving a first consequence of our result, we need to recall the notion of quiver with length appearing in 6, 10.

Let $Q$ be a quiver, and let $\omega=\alpha_{1}^{\varepsilon_{1}} \alpha_{2}^{\varepsilon_{2}} \ldots \alpha_{s}^{\varepsilon_{s}}$ be a walk, where $\alpha_{i} \in Q_{1}$ and $\varepsilon_{i} \in\{-1,1\}$ for all $i$. We call length of $\omega$ the integer defined by $L(\omega)=\sum_{i=1}^{s} L\left(\alpha_{i}^{\varepsilon_{i}}\right)$, where $L\left(\alpha_{i}\right)=1$ and $L\left(\alpha_{i}^{-1}\right)=-1$ for all $i$. Two paths in $Q$ having same source and target are called parallel paths. We say that $Q$ is a quiver with length when any two parallel paths in $Q$ have the same length. Let $\nu, \xi$ be two vertices of the quiver $Q$ with 
length and $n$ an integer. The length $L(\nu, \xi)$ between $\nu$ and $\xi$ is $n$ if there exists a path from $\nu$ to $\xi$ of length $n$.

Proposition 4.1. Let $Q$ be a finite connected acyclic quiver such that the translation quiver $\mathbb{Z} Q$ contains two local slices $\Sigma$ and $\widetilde{\Sigma}$ whose quivers are respectively isomorphic to $Q$ and $Q^{o p}$. If the quiver $Q$ is a quiver with length, then the reflection $S$ induces an anti-automorphism of $\mathbb{Z} Q$.

Proof. We show that the reflection $S: \eta(\Sigma) \longrightarrow \eta(\widetilde{\Sigma})$ constructed in Theorem 3.1 is an anti-isomorphism of quivers. Consider the embedded quiver $\eta(\mathbb{Z} Q)$ defined in Theorem 3.1. Note that all sinks of $\Sigma$ belong to the intersection $\Sigma \cap \widetilde{\Sigma}$ and therefore are mapped to the plane $\mathcal{P}_{0}$. As before $\Sigma$ is decomposed into $k+1$ non-intersecting subquivers $\Sigma^{j}$.

Because the point $A_{j i}$ belongs to the plane $\mathcal{P}_{j}: y=j$, then $j=$ $\rho\left(A_{j i}, \mathcal{P}_{0}\right)$ where $\rho\left(A_{j i}, \mathcal{P}_{0}\right)$ is the Euclidean distance from the point $A_{j l}$ to the plane $\mathcal{P}_{0}$. Note that by the definition of the reflection $S$, we have also $j=\rho\left(S\left(A_{j i}\right), \mathcal{P}_{0}\right)$. Because the quiver $Q$ is a quiver with length, each subquiver $\Sigma^{l}$ of $\Sigma$ is discrete, that is, it contains no arrows. The only arrows of $\Sigma$ are from a subquiver $\Sigma^{j}$ to a subquiver $\Sigma^{j-1}$. Thus in spatial representation of $\eta(\mathbb{Z} Q)$ all arrows are between different planes and therefore are reversed by the reflection $S$, which shows that $S$ is an anti-morphism. To make this reasoning formal, let $\bar{\alpha}_{i l}: A_{j i} \longrightarrow A_{j-1 l}$ be an arrow of $\eta(\Sigma)$. Then the arrow $\left(0, \alpha_{i l}\right)$ : $\left(0, \nu_{j i}\right) \longrightarrow\left(0, \nu_{j-1 l}\right)$ is an arrow of $\Sigma$ and we have $\eta\left(0, \alpha_{i l}\right)=\bar{\alpha}_{i l}$. On the other hand, $\left(-j+1, \alpha_{i l}^{\prime}\right):\left(-j+1, \nu_{j-1 l}\right) \longrightarrow\left(-j, \nu_{j i}\right)$ is an arrow of $\widetilde{\Sigma}$. Since $\eta\left(-j+1, \nu_{j-1 l}\right)=S\left(A_{j-1 l}\right)$ and $\eta\left(-j, \nu_{j i}\right)=S\left(A_{j i}\right)$, then $\eta\left(-j+1, \alpha_{i l}^{\prime}\right)=\bar{\alpha}_{i l}^{\prime}$ is an arrow from $S\left(A_{j-1 l}\right)$ to $S\left(A_{j i}\right)$. Thus $S\left(\bar{\alpha}_{i l}\right)=\bar{\alpha}_{i l}^{\prime}$. Hence $S$ is an anti-isomorphism from the quiver $\Sigma$ to the quiver $\widetilde{\Sigma}$. The symmetry $S$ is extended to an anti-automorphism of the quiver $\mathbb{Z} Q$.

Example 4.1. Let $Q$ be the Dynkin quiver of type $\mathbb{A}_{3}$ given by $1 \stackrel{\alpha_{12}}{\longrightarrow}$ $2 \stackrel{\alpha_{23}}{\longrightarrow} 3$. The quiver $\mathbb{Z} Q$ contains a local slice $\widetilde{\Sigma}$ isomorphic to $Q^{\text {op }}$ which intersects the local slice $\Sigma=(0, Q)$. The quiver $\Sigma$ is given by

$$
(0,1) \stackrel{\left(0, \alpha_{12}\right)}{\longrightarrow}(0,2) \stackrel{\left(0, \alpha_{23}\right)}{\longrightarrow}(0,3)
$$

and the quiver $\widetilde{\Sigma}$ is given by

$$
(0,3) \stackrel{\left(0, \alpha_{23}^{\prime}\right)}{\longrightarrow}(-1,2) \stackrel{\left(-1, \alpha_{12}^{\prime}\right)}{\longrightarrow}(-2,1) .
$$

We set $\eta(0,3)=(0,0,3), \eta(0,2)=(0,1,1)$ and $\eta(0,1)=(0,2,0)$. We have $S(\eta(0,3))=(0,0,3), S(\eta(0,2))=\eta(-1,2)=(-1,-1,1)$ and $S(\eta(0,1))=\eta(-1,1)=(-2,-2,0)$.

We define the anti-morphism $\sigma$ on the points by $\sigma(0,0,3)=(0,0,3)$, $\sigma(0,1,1)=(-1,-1,1)$ and $\sigma(0,2,0)=(-2,-2,0)$; and define on the arrows by $\sigma\left(\eta\left(0, \alpha_{23}\right)\right)=\eta\left(0, \alpha_{23}^{\prime}\right)$ and $\sigma\left(\eta\left(0, \alpha_{12}\right)\right)=\eta\left(-1, \alpha_{12}^{\prime}\right)$. 
The map $\sigma$ is an anti-isomorphism of quivers, which is extended to an anti-automorphism of quiver $\eta(\mathbb{Z} Q)$. The anti-automorphism $\sigma$ is a reflection. Therefore, the reflection $\sigma$ induces an involutive antiautomorphism of the quiver $\mathbb{Z} Q$.

We can now establish the existence of a particular automorphism of the translation quiver $\mathbb{Z} Q$.

Lemma 4.1. Let $Q$ be a finite connected acyclic quiver such that the translation quiver $\mathbb{Z} Q$ contains a local slice whose quiver is isomorphic to $Q^{o p}$. Then there exists an anti-automorphism $\sigma$ of $\mathbb{Z} Q$ such that $\sigma^{2}=1$.

Proof. Let $Q$ be a finite connected acyclic quiver.

If $Q$ is a quiver with length, the reflection $S$ induces an involutive anti-automorphism due to Proposition 4.1.

Suppose that $Q$ is not a quiver with length. According to 10 the quiver $Q$ contains a subquiver of type $\tilde{A}_{p q}$, with $p \neq q$. Let $\Sigma$ and $\widetilde{\Sigma}$ be two local slices of $\mathbb{Z} Q$ whose quivers are respectively isomorphic to $Q$ and $Q^{o p}$. Without loss of generality, we can choose $\Sigma$ and $\widetilde{\Sigma}$ such that $\Sigma_{0} \cap \widetilde{\Sigma}_{0} \neq \emptyset$. Consider the spatial representation $\eta(\mathbb{Z} Q)$ of $\mathbb{Z} Q$ constructed in the proof of Theorem 3.1. In the case without length, there exist planes $\mathcal{P}_{j}$ containing non-discrete quivers. It follows from Theorem 3.1 that $\eta(\Sigma)$ and $\eta(\widetilde{\Sigma})$ are symmetric to each other with respect to the plane $\mathcal{P}_{0}$ of $\mathbb{R}^{3}$ via the reflection $S$. Let $\widetilde{Q}$ be the quiver defined by $\widetilde{\Sigma}=(0, \widetilde{Q})$ and denote by $\tilde{C}^{m}$ the subquiver of $\mathbb{Z} \widetilde{Q}$ of the form $(m, \widetilde{Q})$. Since $\widetilde{\Sigma}$ is a local slice of $\mathbb{Z} Q$, then $\tilde{C}^{m}$ is a local slice of $\mathbb{Z} Q$ for all integer $m$.

By the choice of $\Sigma$ and $\widetilde{\Sigma}$, each sink of $\eta(\Sigma)$ is a source of $\eta(\widetilde{\Sigma})$. Because $\eta(\Sigma)$ is isomorphic to $Q$ and $\eta(\widetilde{\Sigma})$ is isomorphic to $Q^{o p}$, then there exists an anti-isomorphism $\sigma: \eta(\Sigma) \longrightarrow \eta(\widetilde{\Sigma})$ such that every point of $\eta(\Sigma) \cap \eta(\widetilde{\Sigma})$ is invariant under $\sigma$. The anti-isomorphism $\sigma$ induces a permutation $\sigma_{j}$ in $S_{s_{j}}$, where $s_{j}$ is defined as in the proof of the Theorem 3.1. The permutation $\sigma_{j}$ is viewed as the restriction of $S \sigma$ in $\mathcal{P}_{j} \cap \eta\left(\Sigma_{0}\right)$.

We define the map $\bar{\sigma}: \eta(\Sigma) \cup \eta(\widetilde{\Sigma}) \longrightarrow \eta(\Sigma) \cup \eta(\widetilde{\Sigma})$ such that the restriction of $\bar{\sigma}$ on $\eta(\Sigma)$ is $\sigma$ and the restriction of $\bar{\sigma}$ on $\eta(\widetilde{\Sigma})$ is $\sigma^{-1}$. By the definition, the map $\bar{\sigma}$ is an anti-automorphism of the quiver $\eta(\Sigma) \cup \eta(\widetilde{\Sigma})$ which has the following properties:

$\left(p_{1}\right) \bar{\sigma}$ is an involution

$\left(p_{2}\right) \bar{\sigma}$ maps $\mathcal{P}_{j}$ on $\mathcal{P}_{-j}$, with $0 \leq j \leq k$.

We recall that $t_{\vec{u}}$ is the translation in $\mathbb{R}^{3}$ by vector $\vec{u}=(-1,-2,0)$ defined on the step $(b)$ of the proof of the Theorem 3.1. We extend $\bar{\sigma}$ to $\eta(\mathbb{Z} Q)$ by the map $\tilde{\sigma}: \eta(\mathbb{Z} Q) \longrightarrow \eta(\mathbb{Z} Q)$ defined as follows: $\tilde{\sigma}\left(\eta\left(C^{m}\right)\right)=t_{m \vec{u}} \bar{\sigma} t_{m \vec{u}}\left(\eta\left(C^{m}\right)\right)$ for all $m \in \mathbb{Z}$; because $\mathbb{Z} Q \cong \mathbb{Z} \widetilde{Q}$ it is 
equivalent to define $\tilde{\sigma}$ by $\tilde{\sigma}\left(\eta\left(\tilde{C}^{m}\right)\right)=t_{m \vec{u}} \bar{\sigma} t_{m \vec{u}}\left(\eta\left(\tilde{C}^{m}\right)\right)$ for all $m \in \mathbb{Z}$. We have $t_{m \vec{u}}\left(\eta\left(C^{m}\right)\right)=\eta(\Sigma)$ and $t_{-m \vec{u}}\left(\eta\left(C^{-m}\right)\right)=\eta(\widetilde{\Sigma})$ for $m$ nonnegative integer. We set $A_{m i}:=t_{m \vec{u}}\left(A_{j i}\right)$, where $A_{j i}$ is defined as in the proof of the Theorem 3.1 and $\sigma_{j} \in S_{s_{j}}$ is the permutation induced by $\sigma$, with $0 \leq j \leq k$. To make the definition of $\tilde{\sigma}$ more specific, let $A_{m i}$ be a point of $\eta\left(C^{m}\right)$ and $A_{m i}^{\prime}$ the image of $A_{m i}$ under the reflection $S$, we have $\tilde{\sigma}\left(A_{m i}\right)=A_{m \sigma_{j}(i)}^{\prime}=S\left(A_{m \sigma_{j}(i)}\right)$ if $m$ is non-negative integer, and $\tilde{\sigma}\left(A_{m i}\right)=A_{m \sigma_{j}^{-1}(i)}^{\prime}=S\left(A_{m \sigma_{j}^{-1}(i)}\right)$ otherwise, with $0 \leq j \leq k$.

It is enough to define $\tilde{\sigma}$ on the points of $\eta(\mathbb{Z} Q)$, because the definition of $\tilde{\sigma}$ on the arrows is induced by the one on the points. The map $\tilde{\sigma}$ is uniquely determined by its value on the points of $\eta(\mathbb{Z} Q)$ and thus extends in the unique way to an anti-automorphism of the quiver $\eta(\mathbb{Z} Q)$. Hence the extension $\tilde{\sigma}$ of $\sigma$ is an anti-automorphism of the quiver $\eta(\mathbb{Z} Q)$ which maps $C^{m}$ and $\tilde{C}^{-m}$ to each other.

It remains to show that $\tilde{\sigma}$ is an involution. By the definition, it is sufficient to show this just for the points of $\eta(\mathbb{Z} Q)$. Let $A_{m i}$ be a point of $\eta(\mathbb{Z} Q)$, if $m$ is a non-negative integer then

$$
\begin{aligned}
\tilde{\sigma}^{2}\left(A_{m i}\right) & =\tilde{\sigma}\left(A_{m \sigma_{j}(i)}^{\prime}\right) \\
& =A_{m \sigma_{j}^{-1}\left(\sigma_{j}(i)\right)} \\
& =A_{m i} ;
\end{aligned}
$$

if $m$ is a negative integer then

$$
\begin{aligned}
\tilde{\sigma}^{2}\left(A_{m i}\right) & =\tilde{\sigma}\left(A_{m \sigma_{j}^{-1}(i)}^{\prime}\right) \\
& =A_{m \sigma_{j}\left(\sigma_{j}^{-1}(i)\right)} \\
& =A_{m i} .
\end{aligned}
$$

The map $\tilde{\sigma}$ is an involutive anti-automorphism of $\eta(\mathbb{Z} Q)$. Thus $\tilde{\sigma}$ induces an involutive anti-automorphism of $\mathbb{Z} Q$.

In order to illustrate the notations used in the proof of Lemma 4.1 we present an example.

Example 4.2. We consider the quiver $Q$ of the Example 3.1 defined by:

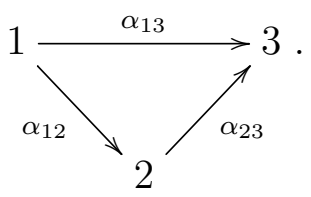

We know that $\eta(0,3)=(0,0,2), \eta(0,2)=(0,1,1)$ and $\eta(0,1)=$ $(0,1,0)$. We have $S(\eta(0,3))=(0,0,2), S(\eta(0,2))=\eta(-1,2)$ and $S(\eta(0,1))=\eta(-1,1)$. We define $\sigma$ by: the arrow $\eta\left(0, \alpha_{12}\right): \eta(0,1) \rightarrow$ $\eta(0,2)$ is mapped to the arrow $\eta\left(-1, \alpha_{12}\right): \eta(-1,1) \rightarrow \eta(-1,2)$, that is $\sigma(\eta(0,1))=\eta(-1,2)$ and $\sigma(\eta(0,2))=\eta(-1,1)$; we have also $\sigma(\eta(0,3))=$ 
$\eta(0,3)$ and $\sigma\left(\eta\left(0, \alpha_{13}\right)\right)=\eta\left(0, \alpha_{23}^{\prime}\right)$ and $\sigma\left(\eta\left(0, \alpha_{23}\right)\right)=\eta\left(0, \alpha_{13}^{\prime}\right)$. The map $\sigma$ is extended to an involutive anti-automorphism of $\eta(\mathbb{Z} Q)$. Hence $\sigma$ induces an involutive anti-automorphism in the transjective component $\Gamma_{\text {tr }} \cong \mathbb{Z} Q$ of the cluster category $\mathcal{C}_{Q}$.

To simplify the notations, let denote by $\sigma$ the anti-automorphism of $\mathbb{Z} Q$ defined above. Let $X=\left\{x_{1}, x_{2}, x_{3}\right\}$ be the set of variables and $\mathcal{A}$ the cluster algebra with initial seed $(X, Q)$. We define the map $f$ from $\mathcal{A}$ to $\mathcal{A}$ by: $f\left(x_{1}\right)=\frac{x_{1}+x_{3}+x_{2} x_{3}^{2}}{x_{1} x_{2}}, f\left(x_{2}\right)=\frac{1+x_{2} x_{3}}{x_{1}}$ and $f\left(x_{3}\right)=x_{3}$. The above data defined an inverse cluster automorphism of $\mathcal{A}$. By observing that $f\left(x_{1}\right)=\frac{1+f\left(x_{2}\right) x_{3}}{x_{2}}$, we have $f^{2}\left(x_{2}\right)=\frac{1+f\left(x_{2}\right) x_{3}}{f\left(x_{1}\right)}=x_{1}$ and $f^{2}\left(x_{1}\right)=\frac{1+f^{2}\left(x_{2}\right) x_{3}}{f\left(x_{2}\right)}=x_{2}$; thus $f$ is an involution.

One can show by using the categorification of the cluster algebra $\mathcal{A}$ as in [7] that the involutive cluster automorphism $f$ is induced by the involutive anti-automorphism $\sigma$.

It has been proved in [3. Theorem 3.11] that, if $\mathcal{A}$ is a cluster algebra with a seed $(X, Q)$ such that $Q$ is a tree then the group of cluster automorphisms is the semidirect product Aut $\mathcal{A}=\mathrm{Aut}^{+} \mathcal{A} \rtimes \mathbb{Z}_{2}$. Moreover this product is not direct. The following result shows that this holds for any finite connected acyclic quiver which is mutation equivalent to its opposite quiver.

Corollary 4.1. Let $Q$ be a finite connected acyclic quiver such that $Q$ and $Q^{o p}$ are mutation equivalent. If $\mathcal{A}$ is a cluster algebra with a seed $(X, Q)$ then the group of cluster automorphisms is the semidirect product $A u t \mathcal{A}=A u t^{+} \mathcal{A} \rtimes \mathbb{Z}_{2}$. This product is usually not direct.

Proof. Let $Q$ be a finite connected acyclic quiver and $\mathcal{A}$ a cluster algebra with a seed $(X, Q)$. We assume without loss of generality that $(X, Q)$ is the initial seed of $\mathcal{A}$, where the quivers $Q$ and $Q^{o p}$ are mutation equivalent. We may assume that $Q$ is not Dynkin, for Dynkin type case we refer to [3. Lemma 3.10]. The transjective component of the cluster category is of the form $\Gamma_{t r} \cong \mathbb{Z} Q$ and $\Gamma_{t r} \cong \mathbb{Z} Q^{o p}$. Then, there exist two local slices $\Sigma$ and $\widetilde{\Sigma}$ of $\Gamma_{t r}$ whose associated quivers are respectively isomorphic to $Q$ and $Q^{o p}$. We may choose $\Sigma$ and $\widetilde{\Sigma}$ such that $\Sigma_{0} \cap \widetilde{\Sigma}_{0} \neq \emptyset$. By 3 , Lemma 3.10] the anti-automorphism $\sigma$ of the Lemma 4.1 induces an inverse cluster automorphism $f_{\sigma}$ of order two. Therefore [3, Corollary 2.12] implies that $\operatorname{Aut} \mathcal{A}=\mathrm{Aut}^{+} \mathcal{A} \rtimes \mathbb{Z}_{2}$. It was proved in [3. Theorem 3.11] that for the particular case where $Q$ is a tree, this product is not direct.

The existence of an involutive anti-automorphism of $\mathbb{Z} \Sigma$ give rise to a characterization of finite connected acyclic quiver $\Sigma$ such that the translation quiver $\mathbb{Z} \Sigma$ contains a local slice whose quiver is isomorphic to $\Sigma^{o p}$. 
Let $Q$ be a finite connected quiver and $R$ a subquiver of $Q$. We call the closure of $R$ and we denote by $\bar{R}$ the smallest full subquiver of $Q$ containing $R$. A symmetric quiver $(Q, \sigma)$ is a pair consisting of a quiver $Q=\left(Q_{0}, Q_{1}, s, t\right)$ and an involution $\sigma: Q_{0} \amalg Q_{1} \longrightarrow Q_{0} \amalg Q_{1}$ with $\sigma\left(Q_{0}\right)=Q_{0}$ and $\sigma\left(Q_{1}\right)=Q_{1}$ such that:

(a) $s(\sigma(\alpha))=\sigma(t(\alpha))$ and $t(\sigma(\alpha))=\sigma(s(\alpha))$ for all $\alpha \in Q_{1}$

(b) If $\sigma(t(\alpha))=s(\alpha)$ then $\sigma(\alpha)=\alpha$.

In other words $Q$ is a symmetric quiver if it has an involutive antiautomorphism. For more details about symmetric quivers, we refer to 15.

Definition 4.1. Let $Q$ be a connected quiver and $\left(Q^{i}\right)_{1 \leq i \leq m}$ a family of full subquivers of $Q$. We say that the family $\left(Q^{i}\right)_{1 \leq i \leq m}$ is a complete decomposition of $Q$ if the following conditions are satisfied:

(C1) $Q_{0}^{i} \cap Q_{0}^{j}=\phi$ if $i \neq j$

(C2) $Q=\overline{Q^{1} \cup Q^{2} \ldots Q^{n}}$.

(C3) The only arrows between two connected components are from $Q^{i}$ to $Q^{i-1}$ for $1 \leq i \leq n-1$

Corollary 4.2. Let $Q$ be a finite connected acyclic quiver such that the translation quiver $\mathbb{Z} Q$ contains a local slice whose quiver is isomorphic to $Q^{o p}$. Then $Q$ possesses a complete decomposition into a family of symmetric subquivers.

Proof. Let $Q$ be a finite connected acyclic quiver, and $\Sigma$ be a local slice in $\mathbb{Z} Q$ whose quiver is isomorphic to $Q$ and $\widetilde{\Sigma}$ be a local slice in $\mathbb{Z} Q$ whose quiver is isomorphic to $Q^{o p}$. We consider the spatial representation of $\mathbb{Z} Q$. Because of Theorem 3.1 and the definition of the spatial representation of $\mathbb{Z} Q$, we assume that $\eta(\Sigma)$ intersects $k+1$ planes $\mathcal{P}_{m}: y=m$, where $0 \leq m \leq k$. We have $\eta\left(\Sigma^{l}\right)=\eta(\Sigma) \cap \mathcal{P}_{l}$, where $\Sigma^{l}$ is the full subquiver of $\eta(\Sigma)$ represented in the plane $\mathcal{P}_{l}: y=l$, via the embedding $\eta$ of Theorem 3.1. By construction, the family $\left(\Sigma^{l}\right)_{0 \leq l \leq k}$ is a complete decomposition of $\Sigma$. It remains to prove that $\Sigma^{l}$ is a symmetric quiver for $0 \leq l \leq k$. We have $\eta\left(\widetilde{\Sigma}^{l}\right)=\eta(\widetilde{\Sigma}) \cap \mathcal{P}_{-l}$, where $\eta\left(\widetilde{\Sigma}^{l}\right)$ is the full subquiver of $\eta(\widetilde{\Sigma})$ represented in the plane $\mathcal{P}_{l}: y=-l$, via the embedding $\eta$ of Theorem 3.1. So the family $\left(\widetilde{\Sigma}^{l}\right)_{0 \leq l \leq k}$ is a complete decomposition of $\widetilde{\Sigma}$.

Since $\widetilde{\Sigma}$ is a local slice of $\mathbb{Z} Q$, then by the Lemma 4.1 , there exists an involutive anti-isomorphism $\sigma: \mathbb{Z} Q \longrightarrow \mathbb{Z} Q$ such that $\sigma(\Sigma)=\widetilde{\Sigma}$.

Because $S\left(\eta\left(\Sigma^{l}\right)\right)$ and $\eta\left(\widetilde{\Sigma}^{l}\right)$ are two full subquivers of $\eta(\widetilde{\Sigma})$ located in the same plane with $\left|S\left(\eta\left(\Sigma^{l}\right)\right)\right|=\left|\eta\left(\widetilde{\Sigma}^{l}\right)\right|$, we deduce that the coincide: $S\left(\eta\left(\Sigma^{l}\right)\right)=\eta\left(\widetilde{\Sigma}^{l}\right)$. Since $S\left(\eta\left(\Sigma^{l}\right)\right) \cong \eta\left(\Sigma^{l}\right)$ and $\eta\left(\widetilde{\Sigma}^{l}\right) \cong\left(\eta\left(\Sigma^{l}\right)\right)^{o p}$, we get that $\eta\left(\Sigma^{l}\right) \cong\left(\eta\left(\Sigma^{l}\right)\right)^{o p}$. Thus the restriction of $\sigma$ on $\eta\left(\Sigma^{l}\right)$ is an involutive anti-automorphism. Hence $\Sigma^{l}$ is a symmetric quiver for all $l$, with $0 \leq l \leq k$. This completes the proof of our assertion. 
Corollary 4.3. Let $Q$ be a finite connected acyclic quiver and $K$ an algebraically closed field. Then $Q$ and $Q^{o p}$ are mutation equivalent if and only if $K Q$ and $K Q^{o p}$ are derived equivalent.

Proof. Let $Q$ be a finite connected acyclic quiver and $K$ an algebraically closed field, $K Q$ the path algebra. Suppose that $Q$ and $Q^{o p}$ are mutation equivalent. Then Corollary 4.1 implies that there exists an inverse automorphism of the cluster algebra $\mathcal{A}$ denoted by $\sigma$. Then, because of 3. Corollary 3.1] $\sigma$ induces a triangle equivalence $\sigma_{\mathcal{D}}: \mathcal{D}^{b}(\bmod K Q) \longrightarrow$ $\overline{\mathcal{D}}^{b}\left(\bmod K Q^{o p}\right)$. Conversely, suppose that $K Q$ and $K Q^{o p}$ are derived equivalent, the cluster category $\mathcal{C}_{Q}$ of the finite connected acyclic quiver $Q$ is an orbit category of $\mathcal{D}^{b}(\bmod K Q)$. It was proved by Keller in 16 , Theorem 1] that the canonical projection $\pi: \mathcal{D}^{b}(\bmod K Q) \longrightarrow \mathcal{C}_{Q}$ is a triangle functor. Therefore $\mathcal{C}_{Q}$ and $\mathcal{C}^{\imath} \vee_{Q}$ are triangulated equivalent. Thus, according to [17], $Q$ and $Q^{o p}$ are mutation equivalent.

\section{REFERENCES}

[1] Assem, I., Brüstle, T., and Schiffler, R. Cluster-tilted algebras without clusters, J. Algebra 324 (2010), no. 9, 2475-2502

[2] I. Assem, G. Dupont and R. Schiffler, On a category of cluster algebras , arXiv:1201.5986v1 [Math.RT] , (2012)

[3] Assem, I. Schiffler, R., and Shramchenko, V. Cluster Automorphisms, Proc. Lond. Math. Soc. (3) 104 (2012), no. 6, 1271-1302.

[4] Assem, I., Simson, D., and Skowroński, A. Elements of the representation theory of associative algebras. Vol. 1. Techniques of representation theory. London Mathematical Society Student Texts, 65. Cambridge University Press, Cambridge, 2006.

[5] Auslander, M., Reiten, I., and Smalø, S.O. Representation theory of Artin algebras. Corrected reprint of the 1995 original. Cambridge Studies in Advanced Mathematics, 36. Cambridge University Press, Cambridge, 1997.

[6] Bongartz, K.; Gabriel, P. Covering spaces in representation-theory., Invent. Math. 65 (1981/82), no. 3, 331-378.

[7] Buan, A.B., Marsh, R.J., Reineke, M. Reiten, I., and Todorov, G. Tilting theory and cluster combinatorics, Adv. Math. 204(2006), 572-618.

[8] Caldero, P., and Chapoton, F. Cluster algebras as Hall algebras of quiver representations, Comment. Math. Helv. 81 (2006), 595-616.

[9] Caldero, P., and Keller, B. From triangulated categories to cluster algebras, Invent. Math. 172 (2008), 169-211.

[10] Chaio, C., Platzeck, M. I., Trepode, S., On the degree of irreducible morphisms., J. Algebra 281 (2004), no. 1, 200-224.

[11] Fomin, S., and Zelevinsky, A. Cluster algebras I: Foundations, J. Amer. Math. Soc. 15 (2002), 497-529

[12] Fomin, S., and Zelevinsky, A. Cluster algebras II: Finite type classification, Ivent. Math. 154 (2003), 63-121

[13] Gekhtman, M., Shapiro, M., and Vainshtein, A. Cluster algebras and Poisson geometry, Moscow Math. J. 3 (2003), 899-934.

[14] Happel, D. Triangulated categories in the representation theory of finitedimensional algebras. London Mathematical Society Lecture Note Series, 119. Cambridge University Press, Cambridge, 1988. 
[15] Derksen, H. and Weyman, J. Generalized quivers associated to reductive groups, Colloquium Math., vol. 94 (2002), no. 2, 151-173.

[16] Keller, B. On triangulated orbit categories, Doc. Math. 10 (2005), 551-581.

[17] Keller, B., and Reiten, I. Acyclic Calabi-Yau categories., Compos. Math. 144 (2008), no. 5, 1332-1348.

[18] Lusztig, S., Canonical bases arising from quantized enveloping algebras., J. Amer. Math. Soc. 3 (1990), no. 2, 447-498.

Département de Mathématiques, Université de Sherbrooke 2500,boul. de l'Université, Sherbrooke, Québec, J1K2R1 Canada .

E-mail address: ndoune.ndoune@usherbrooke.ca 